NASA/TM-97-206241

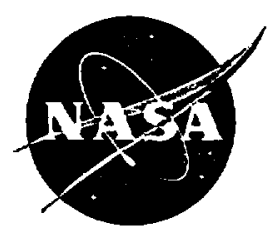

\title{
Breakthrough Propulsion Physics Workshop Preliminary Results
}

Marc G. Millis

Lewis Research Center, Cleveland, Ohio

Prepared for the Space Technology and Applications International Forum cosponsored by NASA, DSWA, U.S. DOE and USAF Albuquerque, New Mexico, 1998

National Aeronautics and

Space Administration

Lewis Research Center 
This report contains preliminary findings, subject to revision as analysis proceeds.

This report is a preprint of a paper intended for presentation at a conference. Because of changes that may be made before formal publication, this preprint is made available with the understanding that it will not be cited or reproduced without the permission of the author.

Available from

NASA Center for Aerospace Information 800 Elkridge Landing Road

Linthicum Heights, MD 21090-2934

Price Code: A03
National Technical Information Service 5287 Port Royal Road Springfield, VA 22100

Price Code: A03 


\title{
BREAKTHROUGH PROPULSION PHYSICS WORKSHOP PRELIMINARY RESULTS
}

\author{
Marc G. Millis \\ NASA Lewis Research Center \\ 21000 Brookpark Rd., MS 60-4, Cleveland, OH 44135 \\ (216) 977-7535, Fax (216) 977-7545 \\ marc.g.millis@lerc.nasa.gov
}

\begin{abstract}
$\underline{\text { Abstract }}$
In August, 1997, a NASA workshop was held to assess the prospects emerging from physics that might lead to creating the ultimate breakthroughs in space transportation: propulsion that requires no propellant mass, attaining the maximum transit speeds physically possible, and breakthrough methods of energy production to power such devices. Because these propulsion goals are presumably far from fruition, a special emphasis was to identify affordable, near-term, and credible research that could make measurable progress toward these propulsion goals. Experiments and theories were discussed regarding the coupling of gravity and electromagnetism, vacuum fluctuation energy, warp drives and wormholes, and superluminal quantum tunneling. Preliminary results of this workshop are presented, along with the status of the Breakthrough Propulsion Physics program that conducted this workshop.
\end{abstract}

\section{INTRODUCTION}

The objective of the NASA Breakthrough Propulsion Physics Program is to make measurable and credible progress toward the seemingly long range goal of creating propulsion breakthroughs. One of the first major milestones of the program was to convene a workshop with established physicists, government researchers and select innovators to jointly examine new theories and phenomena from scientific literature that have reawakened consideration that such breakthroughs may be achievable. Preliminary results of the workshop are presented along with the status of the program. This program, managed by Lewis Research Center, is funded out of the "Advanced Space Transportation Plan" (ASTP) managed by Marshall Space Flight Center (MSFC).

\section{WORKSHOP GOALS}

The purpose of the workshop was to examine emerging physics in the context of seeking propulsion breakthroughs. It is desired to channel the continuing advancements in science toward answering the fundamental questions of how to propel a spacecraft farther, faster, and more efficiently. Specifically, these goals are:

(1) MASS: Discover new propulsion methods that eliminate or dramatically reduce the need for propellant. This implies discovering fundamentally new ways to create motion, presumably by manipulating inertia, gravity, or by any other interactions between matter, fields, and spacetime.

(2) SPEED: Discover how to attain the ultimate achievable transit speeds to dramatically reduce travel times. This implies discovering a means to move a vehicle at or near the actual maximum speed limit for motion through space or through the motion of spacetime itself.

(3) ENERGY: Discover fundamentally new modes of onboard energy generation to power these propulsion devices. This third goal is included since the first two breakthroughs could require breakthroughs in energy generation, and since the physics underlying the propulsion goals is closely linked to energy physics.

To make near-term and measurable progress toward these ambitions, the workshop sought to produce a list of next-step, incremental research approaches. Specifically, this means identifying research tasks that are of relatively short duration and that address the immediate questions raised by the emerging physics and program goals. 


\section{WORKSHOP METHODS}

Three major elements were used at the workshop; a plenary sequence of invited presentations to review emerging physics, a poster paper segment to provide thought-provoking ideas, and breakout sessions to produce a list of candidate next-step research tasks. The first day of the workshop and the morning of the second day featured the invited presentations. This included having opening remarks from Congressman Dennis Kucinich. The breakout sessions followed the invited presentations and were completed by noon of the third day. Summaries of the breakout groups were presented in a plenary session on the afternoon of the third day. The posters were on display throughout the entire workshop.

To keep the number of participants to a manageable number and to provide a constructive mix of physicists, government researchers, and thought-provoking innovators, an invitation-only format was used. Attendance was limited to about 90 participants due to the breakout sessions, where a maximum of 15 participants per each of the six groups was desired. For the overviews of emerging physics, established physicist were invited, including some with constructively pessimistic viewpoints. To foster collaboration between NASA and other government labs, several government researchers were invited. To provoke thought and discussion, poster papers were invited from individuals who had previously submitted materials to NASA on this subject. In total, 84 participants attended the workshop, including 16 from universities; 28 from industry; 11 from government labs including Los Alamos, Oak Ridge, Fermi, Brookhaven and the Air Force Research Labs at Edwards and Kirtland; 17 from NASA including from Lewis, Langley, Marshall, Johnson, and the Jet Propulsion Laboratory; and 12 students.

Since this workshop dealt with seeking breakthroughs in science, it asked participants to be visionary. Admittedly, these breakthroughs may turn out to be impossible, but progress is not made by conceding defeat. For the sake of promoting progress, participants were asked to entertain, for the duration of the workshop, the notion that these breakthroughs are indeed achievable. Simultaneously, however, this workshop looked for sound and tangible research approaches. Therefore, participants were also asked to be credible -- credible progress toward incredible possibilities.

To provide a list of next-step research tasks, the participants were divided into six breakout groups. Each of the three goals mentioned on page 1 were addressed by two of the six groups. Each group was led by a facilitator through a process designed to elicit a large number of creative ideas and then to evolve these ideas into candidate next-step research tasks. This process consisted of the following sequence:

(1) VISION: Participants were asked to assume a priori that the physics breakthroughs needed to create practical interstellar travel were achievable. They were asked to imagine that they were far enough into the future where these breakthroughs have been realized, and were asked to suggest ways that such feats were accomplished.

(2) ISSUES: Participants were asked to identify the critical unknowns and make-or-break issues associated with the ideas from Step-1, and to identify any curious effects (confirmed or unconfirmed) that may support the goals.

(3) NEXT STEPS: Participants were asked to propose the next-step experiments, theoretical analyses, or further theoretical developments that would be needed to resolve the issues identified in Step-2. This included transforming objections into research objectives.

(4) EVALUATION: The ideas from Step-3 were scored using a spreadsheet containing the evaluation criteria shown below. This scoring was more of an experiment of the evaluation process than it was a genuine attempt at scoring the task ideas. As expected, none of the groups were able to score all of the task ideas they generated. However, each group selected a few representative tasks for presentation in the closing plenary session.

- Relevance To Program:

- Directness to Program (Must seek propulsion relevant advances in physics)

- Magnitude of Potential Gains for Goal \#1 (Mass) + Goal \#2 (Speed) + Goal \#3 (Energy)

- Readiness:

- Level of Progress Achieved To Date (using scientific method levels as status metric)

- Testability (ease of empirical testing)

(Note: experiments are considered closer than theory to becoming technology)

- Credibility: (As reflected by peer reviewed scientific literature) 
- Minimum Credibility Criteria for Approaches Not in Peer Reviewed Literature *

- Fits credible data

- Advantageous to propulsion or power goals

- Discriminating test suggested

- Research Task Factors:

- Level of Progress to be Achieved Upon Completion of Task (using scientific method levels as metric)

- Breadth of Work (experiment, theory, and/or comparative study)

- Triage (will it be done anyway or must this program support it?)

- Lineage (will it lead to further relevant advancements?)

- Time Required to Complete Task (reciprocal scoring factor)

- Funding Required (reciprocal scoring factor)

- Probability of Successful Task Completion (credentials and realism of proposal) *

* Due to time limitations during the workshop, these criteria were not used.

\section{INVITED PRESENTATIONS}

The invited presentations, from established physicists, covered many of the relevant areas of emerging physics. The intent of these presentations was to provide credible overviews of where we stand today in physics and introduce the unknowns and unresolved issues. Below is a short synopsis of these presentations in the order that they were presented. Where a related or equivalent work is available in the open literature, a reference is cited.

(1) Lawrence Krauss (Case Western Reserve University, Cleveland OH), "Propellantless Propulsion: The Most Inefficient Way to Fly?": The physics behind manipulating spacetime for propellantless propulsion is reviewed. Even considering the possibilities of new physics, it is shown that such achievements would be probably impossible in practice. (Krauss 1995 and Pfenning 1997)

(2) Harold Puthoff (Institute for Advanced Studies at Austin, TX), "Can the Vacuum be Engineered for Spaceflight Applications?: Overview of Theory and Experiments": Discusses the phenomena of electromagnetic vacuum fluctuations, its empirical evidence, existing applications, and implications for breakthrough space propulsion and power. (Puthoff 1989, Cole 1993, and Haisch 1994)

(3) Raymond Chiao (University of California at Berkeley, CA), and A. Y. Steinberg, "Quantum Optical Studies of Tunneling Times and Superluminality": Presents the experimental methods and results of measuring the tunneling time of a photon to cross a photonic band-gap tunnel barrier, where an effective tunneling speed of 1.7 times the speed of light is measured. The author concludes, however, that information did not travel faster than light. (Chiao 1994)

(4) John G. Cramer (University of Washington, Seattle, WA), "Quantum Nonlocality and Possible Superluminal Effects": Discusses the quantum mechanics of nonlocality, which is often used to speculate about superluminal effects. Examples and unknowns are highlighted, including the transactional interpretation. (Cramer 86)

(5) Ronald J. Koczor, and David Noever (NASA MSFC, Huntsville, AL), "Experiments on the Possible Interaction of Rotating Type II YBCO Ceramic Superconductors and the Local Gravity Field": Presents the status and interim results of experiments being conducted at MSFC to investigate claims of gravity effects in the vicinity of rotating superconductors in strong magnetic fields (Podkletnov 1992). Only static measurements have been completed to date, with inconclusive results (change of less than 2 parts in $10^{8}$ of the normal gravitational acceleration) ( $\mathrm{Li} \mathrm{1997).} \mathrm{Work} \mathrm{continues} \mathrm{toward} \mathrm{measurements}$ with rotating superconductors.

(6) Robert Forward (Forward Unlimited, Clinton, WA), "Apparent Endless Extraction of Energy from the Vacuum by Cyclic Manipulation of Casimir Cavity Dimensions": Proposes a conceptual energy extraction method using cyclic dimensional changes of irregular Casimir cavities. This concept uses data on the energy densities of electromagnetic quantum fluctuations within irregular Casimir cavities, where portions of the data plots are double-valued (Ambjørn 1983 and Forward 1984).

(7) Bernhard Haisch (Lockheed Palo Alto CA) and A. Rueda, "The Zero-Point Field and the NASA Challenge to Create the Space Drive": Proposes that the Newtonian F=ma equation can be derived from Maxwell's equations as applied to the electromagnetic vacuum fluctuations (Haisch 1994). The effective momentum of the vacuum fluctuations is speculated to be a possible basis of propulsion. The author also notes that "negative mass" is incompatible with this theory. 
(8) Alfonso Rueda (California State University, Long Beach, CA) and B. Haisch, "Inertial Mass as Reaction of the Vacuum to Accelerated Motion": Presents further investigations of the theories linking inertia to vacuum fluctuations. (Haisch 1994).

(9) Daniel C. Cole (IBM Microelectronics, Essex Junction, VT), "Calculations on Electromagnetic ZeroPoint Contributions to Mass and Perspectives": Challenges the theories linking inertia to vacuum fluctuations, but supports the idea of vacuum energy exchange (Cole 1993).

(10) Peter W. Milonni (Los Alamos National Labs, Los Alamos NM), "Casimir Effect: Evidence and Implications": Reviews the empirical evidence used to support vacuum fluctuation theories, showing that source fields and macroscopic manifestations of intermolecular forces can also explain observed phenomena. (Milonni 1994).

(11) Hüseyin Yilmaz (Electro-Optics Technology Center, Winchester, MA), "The New Theory of Gravitation and the Fifth Test": Compares the Einstein and Yilmaz theories with data of the gravitational perturbations on Mercury by the other planets. (Yilmaz 92).

(12) Arkady Kheyfets (Dept. of Mathematics, N. Carolina State Univ., Raleigh, NC) and Warner A. Miller, "Hyper-Fast Interstellar Travel via a Modification of Spacetime Geometry": Addresses the key features and obstacles confronting the Alcubierre warp bubble and the Krasnikov tube for hyper-fast travel. Issues include the casual structure, weak and null-energy conditions as well as the violation of chronology protection. It is suggested that quantum gravity may be a more productive avenue to further investigate these possibilities and issues. (Alcubierre 94, Krasnikov 95, and Pfenning 97)

(13) Frank J. Tipler, III (Tulane University, New Orleans, LA), "Ultrarelativistic Rockets and the Ultimate Future of the Universe": Presents a case that no new scientific discoveries are required to traverse the galaxy, provided that antiproton annihilation rockets are used and virtual (computerized) humans do the traveling.

(14) George Miley (University of Illinois, Urbana IL), "Possible Evidence of Anomalous Energy Effects in H/D-Loaded Solids -- Low Energy Nuclear Reactions": Presents empirical evidence of excess energy, radiation emission, and transmutations of elements from experiments involving lattices loaded with deuterium by various methods.

\section{POSTER PAPERS}

To provide imaginative material to help provoke discussion and research ideas, poster papers were solicited for the workshop. Selected posters were on display throughout the workshop, and are listed below, alphabetically by author. In those cases where a related or equivalent work is available in the open literature, a reference is cited. Some posters were based on peer reviewed publications while others were more adventurous and less rigorous. In pioneering work it can be difficult to distinguish between the 'crazy' ideas that will one day evolve into breakthroughs, and the more numerous, genuinely crazy ideas. Even though many ideas proposed for this subject are likely to be incorrect, they can still be useful by provoking other, more viable, ideas. It was in this spirit that ideas beyond the conventional were invited for poster papers.

- D. Alexander (MSE Technology Applications, Inc.), "Replication of an Experiment Which Produced Anomalous Excess Energy."

- C. Asaro (Molecudyne Research), "Special Relativity with Complex Speeds." (Asaro 1996).

- J. D. Baxter (Student, ITT Technical Institute, Salt Lake City), “A Plan For Exceeding The Light Barrier."

- E. W. Davis (National Institute for Discovery Science), "Wormhole Induction Propulsion (WHIP)."

- K. J. Davis (Grad Student, Rutgers University), "Study of M-Theoretic Alcubierre Type Warp Drives."

- S. Dinowitz (Underwriters Laboratories, Inc.), "Michelson-Morley on the Space Shuttle: A Possible Experiment to Test a Field Distortion Theory." (Dinowitz 1996).

- G. F. Erickson (Los Alamos National Lab), "QED Casimir Force Electrical Power Supply."

- R. Forward (Forward Unlimited), "Observational Search for Negative Matter in Intergalactic Voids." (da Costa 1996 and Bondi 1957).

- H. D. Froning Jr. (Flight Unlimited), "Experiments to Explore Space Coupling by Means of Specially Conditioned Electromagnetic Fields." (Froning 1997).

- U. Gat and P. Carpenter (Oak Ridge National Lab), "Nuclear Isomer Decay: A Possibility for Breakthrough Space Propulsion."

- J. G. Hartley (IBM Microelectronics), "Possible Experimental Test of Wheeler-Feynman Absorber Theory." (Heron 1974 and Cramer 1986). 
- N. W. Kantor (Integram), R. D. Eagleton and M. N. Kaplan, "Determination of Existence of the Vacuum Structure." (Eagleton 1983).

- M. N. Kaplan (Motorotor), R. D. Eagleton and N. W. Kantor, "Force Field Propulsion."

- G. Landis (Ohio Aerospace Institute), "An Alcubierre Drive Using Cosmic String."

- J. Maclay (Microfabrications Applications Lab, Univ. of Illinois), M. Serry, B. R. Ilic, P. Neuzil, and D. Czaplewski, "Use of AFM (Automatic Force Microscope) Methods to Measure Variations in Vacuum Energy Density and Vacuum Forces in Microfabricated Structures." (Serry 1995).

- G. L. Matloff (New York Univ.), "The Zero-Point Energy (ZPE) Laser and Interstellar Travel."

- M. G. Millis (NASA Lewis), "The Challenge to Create the Space Drive." (Millis 1997b).

- J. M. Niedra, "Vacuum Fluctuations, Connectivity and Superluminal Physics for Interstellar Travel."

- H. Ringermacher (GE Corp. Research and Development Ctr.), B. Cassenti and D. J. Leopold, "Search for Effects of an Electrostatic Field on Clocks in the Frame of Reference of Charged Particles." (Ringermacher 1994).

- J. J. Roser, "Laboratory Scale Vacuum Energy Extraction Modeled on Weak Nuclear Force Reactions in a Spinning Black Hole System."

- F. Rounds, "Anomalous Weight Behavior in $\mathrm{YBa}_{2} \mathrm{Cu}_{3} \mathrm{O}$, Compounds at Low Temperature."

- D. K. Sen (Grad Student, Dept. of Phys., Univ. of Washington), "Recent Results Concerning the Properties and Structure of the Electromagnetic Vacuum."

- C. Seward (Electron Power Systems, Inc.), "Propulsion and Energy Generation Using the Electron Spiral Toroid." (Seward 1996).

- G. Sobczak (Grad Student, Astronomy Dept., Harvard Univ.), "The Modified Casimir Force in a Uniformly Accelerating Reference Frame and in a Gravitational Field."

- J. V. Vargas and D. G. Torr (Univ. of South Carolina), "Lurking Breakthrough Physics." (Vargas 1991).

- E. L. Wall (Institute for Basic Research), "A First Tangible Step in the Quest for Hyperluminal Space Travel."

- C. K. Whitney (Tufts Univ., Electro-Optics Technology Ctr.), "Challenging the Speed of Light."

- J. F. Woodward (Dept. of Physics, California State Univ.), "Mach's Principle and Impulse Engines: Toward a Viable Physics of Star Trek?" (Woodward 1992 and 1994).

- C. A. Yost (Electric Spacecraft Journal), "Electric Field Propulsion Concepts from Independent Researchers."

- E. Zampino (NASA Lewis), "Can a Hyperspace Really Exist?"

\section{CANDIDATE NEXT-STEP RESEARCH APPROACHES}

Based on the invited presentations, poster papers, and the ideas generated during the breakout sessions, several next-step research approaches were identified and are presented next. These are arranged according to the three program goals and highlight the intriguing phenomena and theories, critical issues, and candidate next-step approaches for each program goal. Regarding the specific task ideas generated during the breakout sessions, about 80 task ideas were collected. These have not yet been fully reviewed, but many are integrated into the discussions below.

\section{Toward Goal 1 - Eliminating Propellant Mass}

It is known that gravity, electromagnetism and spacetime are coupled phenomena. Evidence includes the bending of light, the red-shifting of light, and the slowing of time in a gravitational field as illustrated in Figure 1. This coupling is most prominently described by general relativity (Misner 1973). Given this coupling and our technological proficiency for electromagnetics, it has been speculated that it may become possible to use electromagnetic technology to manipulate inertia, gravity, or spacetime to induce propulsive forces (Millis 1997b). Another phenomena of interest is the Casimir Effect, Figure 2, where closely spaced plates are forced together, presumably by vacuum fluctuations (Lamoreaux 1997). One explanation is that this force is the net radiation pressure of the virtual vacuum fluctuation photons, where the pressure is greater outside the plates than within, since wavelengths larger than the plate separation are excluded. The force is inversely proportional to the 4th power of the distance. Even though this effect can be explained by various theories (Milonni 1994), the idea that the vacuum might create these forces leads to speculations that an asymmetric vacuum effect, if possible, could lead to a propulsive effect (Millis 1997b). There are many unsolved issues regarding these speculations, including whether these phenomena can lead to controllable net-force effects and whether such effects can be created, even in principle, without violating conservation of momentum and energy (Millis 1997b). 


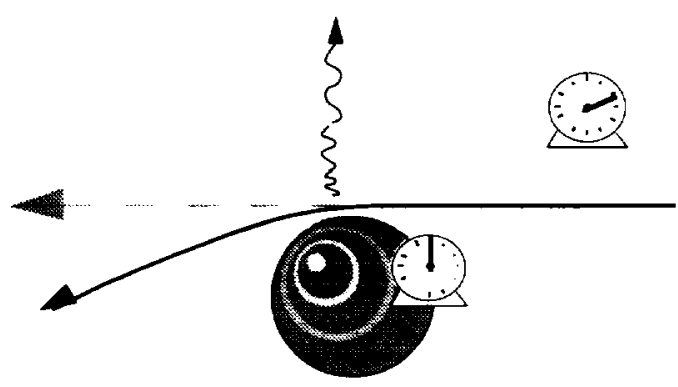

FIGURE 1. Coupling of Gravity, Spacetime, and Electromagnetism.

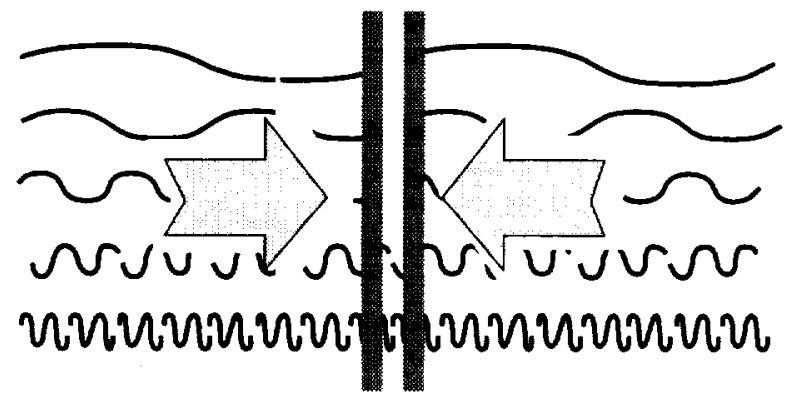

FIGURE 2. The Casimir Effect

Although it is presently unknown if such propellantless propulsion can be achieved, several theories have emerged that provide additional research paths. It should be noted that all of these theories are too new to have either been confirmed or discounted, but their potential utility warrants consideration. This includes negative mass propulsion (Bondi 1957), theories that suggest that inertia and gravity are affected by vacuum fluctuations (Puthoff 1989 and Haisch 1994), and numerous other theories about the coupling between matter, electromagnetism, and spacetime (Dinowitz 1996, Froning 1997, Ringermacher 1994, Vargas 1991, Woodward, 1992, and Yilmaz 1992). Another recent development, which has yet to be credibly confirmed or discounted, is where anomalous weight changes are observed over spinning superconductors (Podkletnov 1992).

During the workshop these possibilities were discussed with an emphasis on experimental verification. A poster by Forward suggested a search for evidence of negative mass based on recent astronomical data (da Costa 1996). The posters of Dinowitz, Froning, Ringermacher, and Woodward all offered experiments to test their theories. Several experiments were suggested to test the theories linking inertia to vacuum fluctuations, including experiments described in existing literature (Forward 1996). And interest was expressed in continuing the experiments to test the claims of weight changes over spinning superconductors (Li 1997).

\section{Toward Goal 2 - Achieving the Ultimate Transit Speed}

Special relativity states that the speed of light is an upper limit for the motion of matter through spacetime. Recently, however, theories using the formalism of general relativity have suggested that this limit can be circumvented by altering spacetime itself. This includes "wormhole" and "warp drive" theories. A wormhole is a shortcut created through spacetime (Morris 1988 and Visser 1995), as illustrated in Figure 3, where a region of spacetime is warped to create a shorter path between two points. A warp drive involves the expansion and contraction of spacetime to propel a region of spacetime faster than light (Alcubierre 1994). Figure 4 illustrates the Alcubierre warp drive, showing the opposing regions of expanding and contracting spacetime that propel the center region.

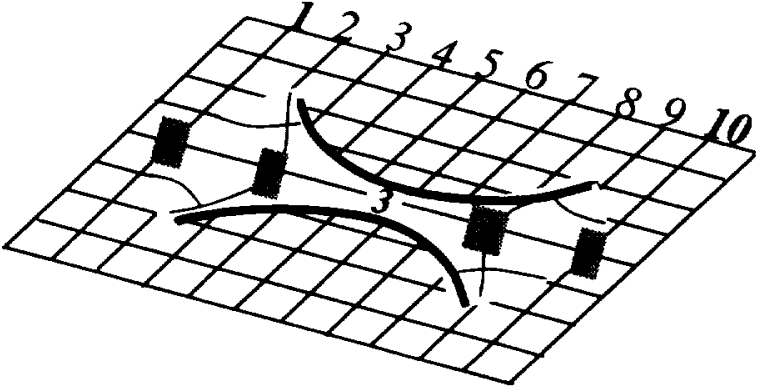

FIGURE 3. Wormholes - Spacetime Shortcuts.

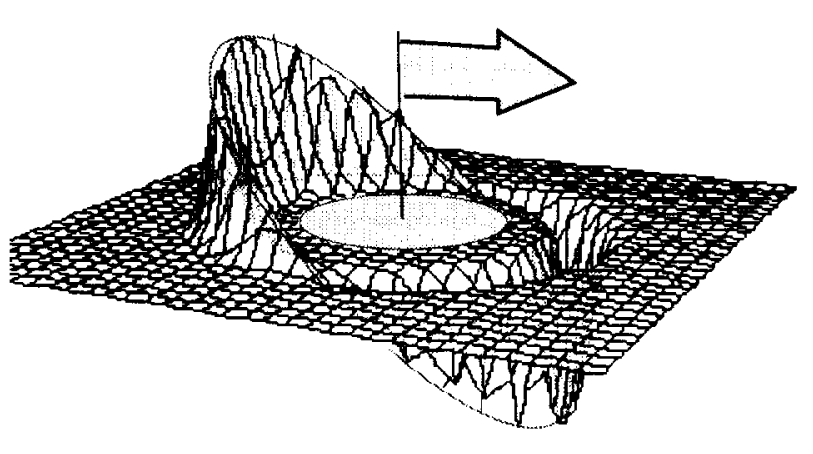

FIGURE 4. The Alcubierre Warp Drive 
It has also been suggested that the light speed limit could be exceeded if velocities could take on imaginary values (Asaro 1996). In addition, there are theories for "nonlocality" from quantum physics that suggest potentially superluminal effects (Cramer 1986). These theories not only present challenging physics problems, but are intriguing from the point of view of future space travel. Do these theories represent genuinely possible physical effects, or are they merely mathematical curiosities?

In addition to theories, there are some intriguing experimental effects. Photons have been measured to tunnel across a photonic band-gap barrier at 1.7 times the speed of light (Chiao 1994). Even though the author concludes that information did not travel faster than light, the results are intriguing. During the workshop several suggestions were made to conduct similar experiments using matter rather than photons to unambiguously test the information transfer rate. In addition, recent experiments of the rest mass of the electron antineutrino have measured an imaginary value (Stoeffl 1995). Even though this result is attributed to possible errors, an imaginary mass value is a signature characteristic of a tachyon. Tachyons are hypothetical faster-than-light particles. In the workshop it was suggested to revisit this and other similar data to determine if this can be credibly interpreted as evidence of tachyons. It was also pointed out that other experiments have been suggested to search for evidence of tachyons (Chiao 1996).

The notion of faster-than-light travel evokes many critical issues. These were summarized in the presentation by Kheyfets. Issues include causality violations, the requirement for negative energy, and the requirement for enormous energy densities to create the superluminal effects. Suggestions were made during the workshop for a number of theoretical approaches to address these issues, including the use of quantum gravity to study the wormhole and warp drive concepts.

\section{Toward Goal 3 - Discovering New Modes of Energy Production}

Since the first two breakthroughs could require breakthroughs in energy generation, and since the physics underlying the propulsion goals is closely linked to energy physics, it is also of interest to discover fundamentally new modes of energy generation. The principle phenomena of interest for this category is, again, the vacuum fluctuations. It has been theorized that this energy can be extracted without violating conservation of energy or any thermodynamic laws (Forward 1984, Cole 1993). It is still unknown if this vacuum energy exists as predicted, how much energy might be available to extract, and what the secondary consequences would be of extracting vacuum energy.

At the workshop, the techniques described in the posters of Maclay (Serry 1995), Erickson, and Sen were suggested to investigate the energy extraction concepts described in the poster of Erickson and by the presentation by Forward. These techniques involve the use of micromechanical structures. Not only are micromechanical structures an emerging technology, but the dimensions of such structures are similar to the dimensions required for Casimir effects. Also, should any viable device be engineered, these methods hold promise for high-volume manufacturing.

On a more conventional vein, ideas were raised at the workshop by Tipler and others for seeking alternative methods of antimatter production. Also, the poster by Seward presented a novel energy storage device involving toroidal plasmas (Seward 1996).

\section{PROGRAM STATUS AND NEXT STEPS}

The Breakthrough Propulsion Physics Program was established in 1996 as part of the "Advanced Space Transportation Plan" (ASTP) managed by MSFC (Millis 1997a). A government Steering Group containing volunteers from various NASA centers, DoD and DoE laboratories and led by the Lewis Research Center, has been established to guide this program. This includes the development of the research solicitation and selection criteria used during the workshop, and which are being refined for future evaluations of research proposals.

The first major milestone of the program was this kick-off workshop. A publicly available Conference Proceedings is being assembled to fully document the results. The list of task ideas generated during the workshop will be used to advocate for funding to support this research. 
Once funded, this program plans to use an annual "NASA Research Announcement" (NRA) to solicit and support research tasks. This solicitation will be open to academia, industry, government labs, and NASA centers. Selection will be via a peer review process led by the Steering Group and using the prioritization criteria to provide an initial ranking. Because it is too early to focus on a given approach, it is anticipated that multiple, different approaches will be supported from the top ranking candidates. Proposed tasks should be of relatively short duration (1-3yrs), modest cost $(\$ 50$ to $\$ 150 \mathrm{~K})$, and traceable to at least one of the three program goals.

The next step of the program, with or without funding, is to reopen participation to the broader set of government, university and industry researchers. An internet site has been established as a first step to begin this collaboration (http://www.lerc.nasa.gov/WWW/bpp/). Also, a limited access site is envisioned to contain works in progress, more in-depth annotated bibliographies, and allow on-line discussions. Access will be limited to a Contributor Network selected by the Steering Group. The process for nominating and selecting Contributor Network members has not yet been established.

\section{CONCLUSIONS}

New theories and laboratory-scale effects have emerged in the scientific literature which provide new approaches to seeking major propulsion breakthroughs. During the recent workshop, many of these new approaches were reviewed, and about 80 specific research task ideas were generated for making progress toward propulsion breakthroughs. A peer review system has been drafted that can be used to rank these and other future proposals.

\section{Acknowledgments}

Special thanks is owed to the Lewis Research Center volunteers who helped make this workshop a success: Obasi Akan, Sheila Bailey, Michael Binder, David Chato, Dane Elliott-Lewis, Cynthia Forman, James Giomini, Jon Goldsby, Scott Graham, Al Juhasz, Geoffrey Landis, Grace Scales, Gary Scott Williamson, Natalie Woods, Ed Zampino, and especially to Joe Hemminger for arranging the equipment and volunteers for the breakout sessions. Special thanks is also owed to the volunteer members of the BPP Steering Group, especially to Frank Mead. In addition, thanks is owed to the Honorable Dennis Kucinich for his opening remarks, and to the NYMA Inc. staff for smoothly handling the workshop logistics; Dr. Richard Ziegfeld, Linda Oliver, and John Toma.

\section{References}

Ambjørn, J. and Wolfram, S. (1983) "Properties of the Vacuum, 1. Mechanical and Thermodynamic, and Properties of the Vacuum, 2. Electrodynamic," Annals of Physics, 147:1-56.

Alcubierre, M. (1994) "The Warp Drive: Hyper-fast Travel Within General Relativity," Classical and Quantum Gravity, 11:L73-L77.

Asaro, C. (1996) "Complex Speeds and Special Relativity," Am. J. Phys., 64(4):412-429.

Bondi, H. (1957) "Negative Mass in General Relativity," Reviews of Modern Physics, 29:423-428.

Chiao, R. Y., Steinberg, A. M., and Kwiat, P. G. (1994) "The Photonic Tunneling Time and the Superluminal Propagation of Wave Packets," Proc. of the Adriatico Workshop on Quantum Interferometry, DeMartini, Denardo, and Zeilinger, eds., World Scientific, Singapore, p. 258.

Chiao, R. Y., Kozhekin, A. E., and Kurizki G. (1996) "Tachyonlike Excitations in Inverted Two-Level Media," Phys. Rev. Lett. 77:1254

Cole, D. and Puthoff, H. (1993) "Extracting Energy and Heat from the Vacuum," Phys Rev E, 48:1562-1565. 
Cramer, J. G. (1986) "The Transactional Interpretation of Quantum Mechanics," Reviews of Modern Physics, A. Phys. Soc., 58:647-688.

da Costa, L. N., Freudling, W., Wegner, G., Giovanelli, R., Haynes, M. P., and Salzer, J. J. (1996) "The Mass Distribution in the Nearby Universe," Astrophysical Journal Letters, 468: L5-L8 and Plate L1

Dinowitz, S. (1996) "Field Distortion Theory," Physics Essays, 9:393-418.

Eagleton, R. D. and Kaplan, M. N. (1983) "The Radial Magnetic Field Homopolar Motor," Am. J. Phys, $56: 858$.

Forward, R. L. (1984) "Extracting Electrical Energy from the Vacuum by Cohesion of Charged Foliated Conductors," Physical Review B, 15 AUG. 1984 B30:1700-1702.

Forward, R. L. (1996) "Mass Modification Experiment Definition Study," Report \# PL-TR-96-3004, Phillips Lab, Edwards AFB, CA.

Froning, H. D. and Barrett, T. W. (1997) "Inertial Reduction and Possible Impulsion by Conditioning Electromagnetic Fields," AIAA 97-3170, 33rd AIAA/ASME/SAE/ASEE Joint Propulsion Conference.

Haisch, B., Rueda, A., and Puthoff, H. E. (1994) "Inertia as a Zero-Point Field Lorentz Force," Physical Review A, 49:678-694.

Heron, M. L. and Pegg, D. T. (1974) “A Proposed Experiment on Absorber Theory,” J. Phys A, 7:1965-1969.

Krasnikov, S. V. (1995) “Hyper-Fast Interstellar Travel in General Relativity,” gr-qc, 9511068.

Krauss, L. M. (1995) The Physics of Star Trek, Basic Books, NY.

Lamoreaux, S. K. (1997) "Demonstration of the Casimir Force in the 0.6 to $6 \mu \mathrm{m}$ Range," Phys. Rev. Letters, 78:5-8.

Li, N., Noever, D., Robertson, T., Koczor, R., and Brantley, W. (1997) "Static Test for a Gravitational Force Coupled to Type II YBCO Superconductors," Physica C, 281:260-267.

Millis, M. (1997a) "Breakthrough Propulsion Physics Research Program," NASA TM 107381, Lewis Research Center. Also at: http://www.lerc.nasa.gov/WWW/bpp/.

Millis, M. (1997b) “Challenge to Create the Space Drive," Journal of Propulsion and Power, 13:577-582.

Milonni, P. W. (1994) The Quantum Vacuum, Academic Press, San Diego, CA.

Misner C. W., Thorne, K. W., and Wheeler, J. A. (1973) Gravitation, W. H. Freeman and Company, NY.

Morris, M. and Thorne, K. (1988) "Wormholes in Spacetime and Their Use for Interstellar Travel: A Tool for Teaching General Relativity," American Journal of Physics, 56:395-412.

Pfenning, M., Ford, L. (1997) “The Unphysical Nature of Warp Drive,” gr-qc, 9702026.

Podkletnov, E. and Nieminen, R. (1992) “A Possibility of Gravitational Force Shielding by $\mathrm{Bulk}^{\mathrm{YBa}_{2} \mathrm{Cu}_{3}}$ $\mathrm{O}_{7-\mathrm{x}}$ Superconductor," Physica C, C203:441-444.

Puthoff, H. E. (1989) “Gravity as a zero-point-fluctuation force,” Phys Rev A, 39:2333-2342.

Ringermacher, H. (1994) “An Electrodynamic Connection," Classical and Quantum Gravity, 11:2383-2394. 
Serry, F. M., Walliser, D., Maclay, G. J. (1995) “The Anharmonic Casimir Oscillator," J. Microelectromechanical Systems, 4:193.

Seward, D. C. (1996) "Energy Storage System," US Patent 5,589,727.

Stoeffl, W. and Decman D. J. (1995) "Anomalous Structure in the Beta Decay of Gaseous Molecular Tritium," Physical Review Letters, 75:3237-3240.

Vargas, J. (1991) "On the Geometrization of Electrodynamics," Foundations of Physics, 21:379-401.

Visser, M. (1995) Lorentzian Wormholes - From Einstein to Hawking, AIP Press, Woodbury, NY.

Woodward, J. F. (1992) "A Stationary Apparent Weight Shift From a Transient Machian Mass Fluctuation," Foundations of Physics Letters, 5:425-442.

Woodward, J. F. (1994) "Method for Transiently Altering the Mass of an Object to Facilitate Their Transport or Change their Stationary Apparent Weights," US Patent 5,280,864.

Yilmaz, H. (1992), "Toward a Field Theory of Gravitation," Il Nuovo Cimento, 107B:941-960. 



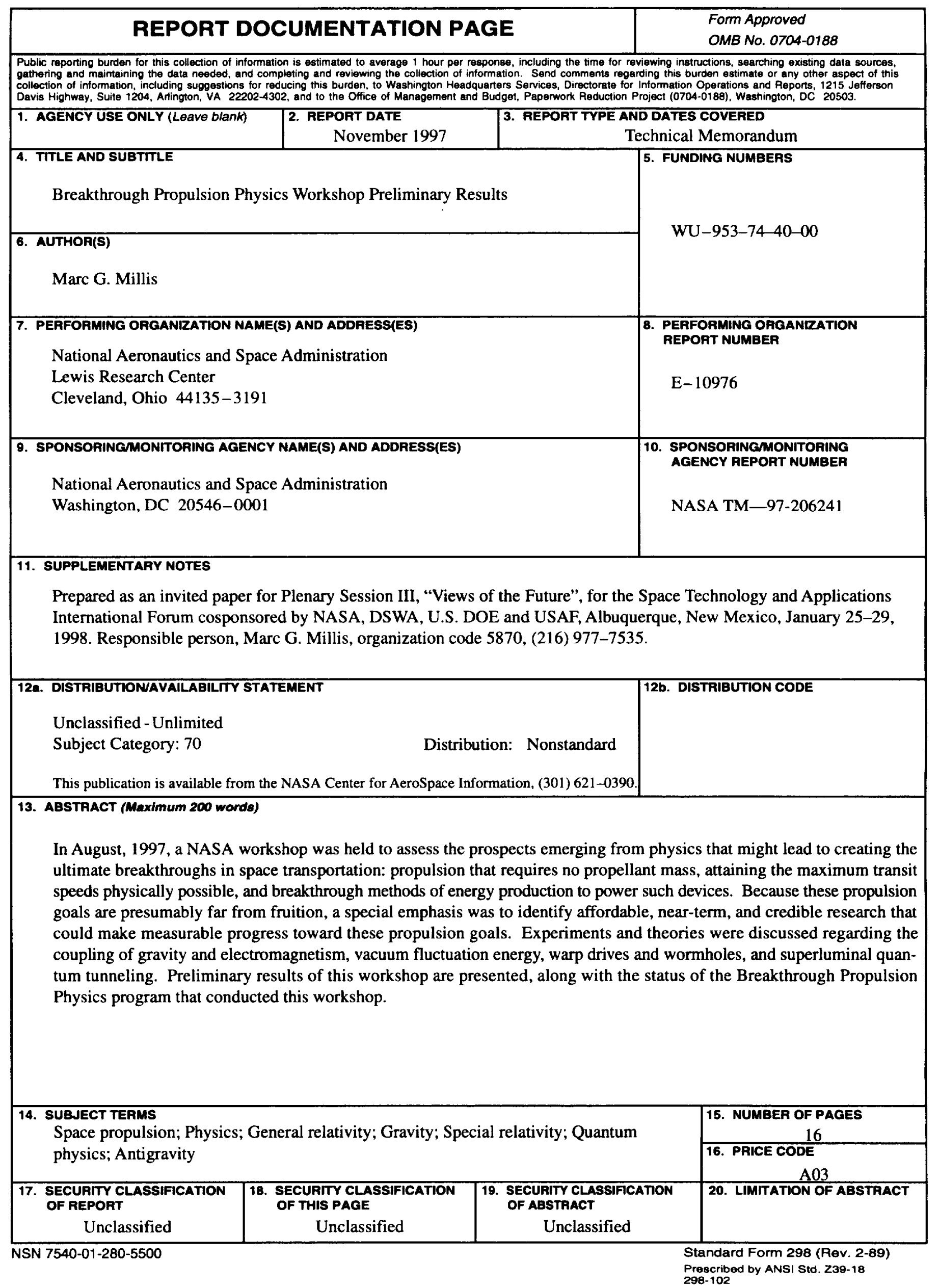

